\title{
Hirudin, a thrombin inhibitor, attenuates TGF- $\beta$-induced fibrosis in renal proximal tubular epithelial cells by inhibition of protease-activated receptor 1 expression via S1P/S1PR2/S1PR3 signaling
}

\author{
QIANG LIN ${ }^{1}$, CHUNLI LONG ${ }^{2}$, ZHENGANG WANG ${ }^{3}$, RONGHUI WANG ${ }^{4}$, \\ WEI SHI ${ }^{1}$, JIWEI QIU ${ }^{2}$, JUNLIN $\mathrm{MO}^{2}$ and YONGXIANG XIE ${ }^{1}$
}

\begin{abstract}
${ }^{1}$ Department of Nephrology, The First Affiliated Hospital of Guangxi University of Chinese Medicine, Nanning, Guangxi 530023; ${ }^{2}$ Department of Health Maintenance, Faculty of Preclinical Medicine, Guangxi University of Chinese Medicine, Nanning, Guangxi 530001; Departments of ${ }^{3}$ Endocrinology and ${ }^{4}$ Critical Care Medicine, The First Affiliated Hospital of Guangxi University of Chinese Medicine, Nanning, Guangxi 530023, P.R. China
\end{abstract}

Received November 26, 2020; Accepted June 16, 2021

DOI: 10.3892/etm.2021.10924

\begin{abstract}
Renal interstitial fibrosis (RIF) is the final common outcome of numerous chronic kidney diseases, contributing to end-stage renal disease. Hirudin, a thrombin inhibitor, has attracted increased attention as a potential treatment approach for renal fibrosis. The present study aimed to investigate the molecular mechanism underlying the effect of hirudin on fibrosis in renal proximal tubular epithelial cells. An in vivo mouse RIF model established using unilateral ureteral obstruction (UUO) and an in vitro of RIF using the renal tubular epithelial cell line HK-2 treated with TGF- $\beta$ were used. Expressions of sphingosine-1-phosphate (S1P) receptors (S1PR)1-4 and protease-activated receptor 1 (PAR1) were measured by reverse transcription-quantitative PCR and western blotting in mice with UUO and TGF- $\beta$ induced HK-2 cells. Western blotting was used to detect the expression of $\mathrm{N}$-cadherin, Slug, E-cadherin, Collagen IV, fibronectin, MMP9 and monocyte chemoattractant protein-1. Immunofluorescence staining was conducted to measure $\alpha$-SMA level expression. The results demonstrated that the expression levels of S1PR1, S1PR2, S1PR3, S1PR4 and PAR1 were upregulated in both TGF- $\beta$-induced HK- 2 cells and renal tissues from mice with unilateral ureteral ligation. Notably, hirudin inhibited TGF- $\beta$-induced PAR1, S1PR2 and S1PR3 upregulation in
\end{abstract}

Correspondence to: Professor Yongxiang Xie, Department of Nephrology, The First Affiliated Hospital of Guangxi University of Chinese Medicine, 89-9 Dongge Road, Qingxiu, Nanning, Guangxi 530023, P.R. China

E-mail:weissw99@163.com

Key words: hirudin, renal interstitial fibrosis, TGF- $\beta$, proteaseactivated receptor 1, sphingosine 1-phosphate/sphingosine 1-phosphate receptor signaling both HK-2 cells and renal tissues. Additionally, the inhibition of S1PR2 and S1PR3 resulted in PAR1 downregulation. Furthermore, treatment with S1P and PAR1 agonists abolished the effect of hirudin on the expression of EMT, fibrosis-related proteins and monocyte chemoattractant protein 1 . In conclusion, hirudin attenuated TGF- $\beta$-induced fibrosis in proximal renal tubular epithelial HK-2 cells by inhibiting PAR1 expression via the S1P/S1PR2/S1PR3 signaling pathway. Therefore, hirudin may be considered as a promising therapeutic agent for RIF.

\section{Introduction}

Renal fibrosis is recognized as the hallmark of the majority of progressive renal diseases, including interstitial fibrosis and glomerular sclerosis $(1,2)$. Renal interstitial fibrosis (RIF) is frequently the final outcome of several chronic kidney diseases (CKDs), and is known as one of the fundamental pathological changes leading to end-stage renal disease (3-5). Due to their high prevalence, CKDs and renal fibrosis affect $~ 10 \%$ of the global population, particularly elderly individuals $(6,7)$. Since the majority of renal diseases contribute to renal fibrosis, there is considerable interest in investigating their underlying etiology to prevent or reverse the aforementioned pathological changes.

The progression of CKD is characterized by the loss of renal cells and their replacement by extracellular matrix $(\mathrm{ECM})$. A previous study demonstrated that the excessive deposition and modification of the ECM in the renal parenchyma lead to glomerulosclerosis and tubulointerstitial fibrosis (8). Unilateral ureteral ligation (UUO) is one of the classical models of renal tubulointerstitial fibrosis, which affects the collecting ducts and results in extensive proximal tubular degeneration (9). Moreover, growing evidence has indicated that the proximal tubular injury caused by UUO is associated with RIF (10). Additionally, several molecules have been found to be involved in the progression of RIF, including 
TGF- $\beta(3,11,12)$. However, there are currently no effective treatment approaches for preventing the onset and development of renal fibrosis. Therefore, investigating the cellular and molecular mechanisms underlying RIF progression is of great importance for its reversal or elimination.

Hirudin, a thrombin inhibitor, is extracted from the salivary gland of the medicinal leech Hirudo medicinalis (13). Hirudin has gained increasing interest as a potential treatment for renal fibrosis, due to its relatively safe and inexpensive components that benefit kidney health (14). Our previous study showed that hirudin inhibited fibrosis in both renal tissues and renal tubular epithelial cells via attenuation of inflammation, regulating the expression of fibrosis- and epithelial-mesenchymal transition (EMT)-related proteins, and reducing the apoptotic rate of renal tubular epithelial cells (15). However, the molecular mechanism underlying the effect of hirudin on RIF has not yet been elucidated.

The non-hemostatic cellular effects of thrombin are mediated by the activation of G-protein-coupled receptors, known as protease-activated receptors (PARs). These receptors are expressed on the surface of endothelial cells and numerous other cell types, including proximal tubular epithelial cells (14). HK-2 cells express PAR1 (16); therefore, the effect of hirudin on renal fibrosis may be triggered by protease-activated receptor 1 (PAR1) signaling. Furthermore, another study demonstrated that thrombin enhanced the activation of glomerular endothelial cells via the sphingosine-1-phosphate (S1P)/sphingosine-1-phosphate receptor 3 (S1PR3) signaling pathway (17), and a further study showed that S1P upregulated the expression of PAR1 and PAR4 (18). The aim of the present study was to investigate the effects of hirudin on TGF- $\beta$-induced fibrosis in renal proximal tubular epithelial cells, as well as its underlying molecular mechanism.

\section{Materials and methods}

Animal model. All animal experiments were approved by the Guangxi University of Chinese Medicine Institutional Animal Ethics and Welfare Committee (approval no. DW20190107-013), and were carried out according to the institutional guidelines for the care and use of laboratory animals. In total, 60 male balb/c mice aged 12 weeks ( $25 \pm 3 \mathrm{~g}$ weight) were obtained from the Laboratory Animal Center of Peking University Health Science Center (license no. DW20190107-013; Beijing, China). The animals were kept under standard conditions at a room temperature of $25 \pm 2{ }^{\circ} \mathrm{C}$ with $60 \%$ humidity with 12 -h light-dark cycle and allowed free access to feed and tap water. After 2 weeks of adaptive feeding, the mice were randomly divided into four groups $(n=15)$, including the control, UUO, UUO + hirudin $(10 \mathrm{mg} / \mathrm{kg})$ and UUO + hirudin $(15 \mathrm{mg} / \mathrm{kg})$ groups. The UUO mouse model was established as previously described (12). Briefly, the mice were anaesthetized with an intraperitoneal injection of $4 \%$ chloral hydrate $(370 \mathrm{mg} / \mathrm{kg})$. No signs of peritonitis, pain or discomfort were observed after anesthesia. Following the establishment of the UUO model, two thirds of the UUO mice were administrated with 10 or $15 \mathrm{mg} / \mathrm{kg}$ hirudin on a daily basis. On day 21 after surgery, the mice were sacrificed by cervical dislocation and the kidneys were harvested.
Cell culture and treatment. Immortalized proximal tubular epithelial cells (HK-2) were obtained from the American Type Culture Collection, and maintained in $90 \%$ high-glucose DMEM (Thermo Fisher Scientific, Inc.) supplemented with $10 \% \mathrm{FBS}$ at $37^{\circ} \mathrm{C}\left(5 \% \mathrm{CO}_{2}\right)$ in a humidified atmosphere. Subsequently, the cells were cultured with TGF- $\beta(5 \mathrm{ng} / \mathrm{ml}$; Sigma-Aldrich; Merck KGaA) for $48 \mathrm{~h}$ at $37^{\circ} \mathrm{C}$ to establish an in vitro RIF cell model. In addition, the HK-2 cells were treated with hirudin $(0.5$ or $1 \mathrm{mg} / \mathrm{ml}$; Sigma-Aldrich; Merck $\mathrm{KGaA}$ ) in the presence or absence of $100 \mathrm{nM}$ of S1P for $48 \mathrm{~h}$ at $37^{\circ} \mathrm{C}$ or $50 \mu \mathrm{M}$ of TFL (a PAR1 agonist; Sigma-Aldrich; Merck KGaA) for $2 \mathrm{~h}$ at $37^{\circ} \mathrm{C}$.Cells were also treated with $5 \mathrm{ng} / \mathrm{ml}$ TGF- $\beta$, followed by $10 \mu \mathrm{M}$ JTE-013 (a S1PR2 antagonist; Sigma-Aldrich; Merck KGaA) for $2 \mathrm{~h}$ at $37^{\circ} \mathrm{C}$ or $1 \mu \mathrm{M}$ TY52156 (a S1PR3 antagonist; Sigma-Aldrich; Merck KGaA) for $2 \mathrm{~h}$ at $37^{\circ} \mathrm{C}$.

Reverse transcription-quantitative PCR (RT-qPCR). Total RNA was extracted from HK-2 cells using a TRIzol ${ }^{\circledR}$ reagent kit (Thermo Fisher Scientific, Inc.) according to the manufacturer's instructions. RNA was reverse transcribed into cDNA using the PrimeScript ${ }^{\circledR}$ RT reagent kit (Takara Bio, Inc.) and the reaction was incubated at $25^{\circ} \mathrm{C}$ for $5 \mathrm{~min}, 42^{\circ} \mathrm{C}$ for $30 \mathrm{~min}$, $85^{\circ} \mathrm{C}$ for $5 \mathrm{~min}$, and then kept at $4^{\circ} \mathrm{C}$ for $5 \mathrm{~min}$. Subsequently, qPCR was conducted using the SYBR Premix EX Taq ${ }^{\mathrm{TM}}$ II kit (Takara Bio, Inc.) on the PRISM 7500 system (Applied Biosystems; Thermo Fisher Scientific, Inc.). The thermocycling conditions were $95^{\circ} \mathrm{C}$ for $3 \mathrm{~min}$, followed by 35 cycles of denaturation at $95^{\circ} \mathrm{C}$ for $30 \mathrm{sec}$, annealing at $60^{\circ} \mathrm{C}$ for $30 \mathrm{sec}$ and extension at $72^{\circ} \mathrm{C}$ for $1 \mathrm{~min}$. A final extension step at $72^{\circ} \mathrm{C}$ for $7 \mathrm{~min}$. The relative mRNA expression levels of the target genes were determined using the $2^{-\Delta \Delta C q}$ method (19), and normalized to that of GAPDH. The primer sequences were as follows: S1PR1 forward, 5'-TCTGCTCCTGCTTTCCA TCG-3' and reverse, 5'-AGGATGTCACAGGTCTTCGC-3'; S1PR2 forward, 5'-CCTTCGTGGCCAACACCTTA-3' and reverse, 5'-TGTCACTGCCGTAGAGCTTG-3'; S1PR3 forward, 5'-ACC GCGTGTTCCTTCTGATT-3' and reverse, 5'-TTGACCAGG CAGTAGATGCG-3'; S1PR4 forward, 5'-GTGTATGGCTG CATCGGTCT-3' and reverse, 5'-CCACTAGGATGAGGGCG AAG-3';PAR1 forward,5'-TGTGAACTGATCATGTTTATG-3' and reverse, 5'-TTCGTAAGATAAGAGATATGT-3'; monocyte chemoattractant protein 1 (MCP-1), forward, 5'-GCTCAT AGCAGCCACCTTCATTC-3' and reverse, 5'-GTCTTCGGA GTTTGGGTTTGC-3'; and GAPDH forward, 5'-GGGAGC CAAAAGGGTCATCATCT-3' and reverse, 5'-GACGCCTGC TTCACCACCTTCTTG-3'.

Western blot analysis. The renal tissue samples and HK-2 cells were lysed with RIPA buffer (Cell Signaling Technology, Inc.) supplemented with protease inhibitors. A BCA Protein Assay kit (Beijing Dingguo Changsheng Biotechnology Co., Ltd.) was used for protein quantification, according to the manufacturer's instructions. A total of $30 \mu \mathrm{g}$ protein per lane was then separated by $10 \%$ SDS-PAGE (Bio-Rad Laboratories, Inc.) and transferred onto a PVDF membrane (EMD Millipore). Following blocking with 5\% non-fat milk for $1 \mathrm{~h}$ at room temperature, the membrane was incubated with specific primary antibodies against S1PR1 (1:1,000; cat. no. ab242085; Abcam), S1PR2 (1:1,000; cat. no. ab235919; Abcam), S1PR3 
A

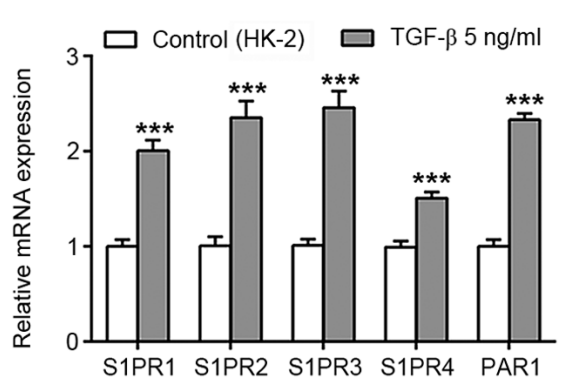

C

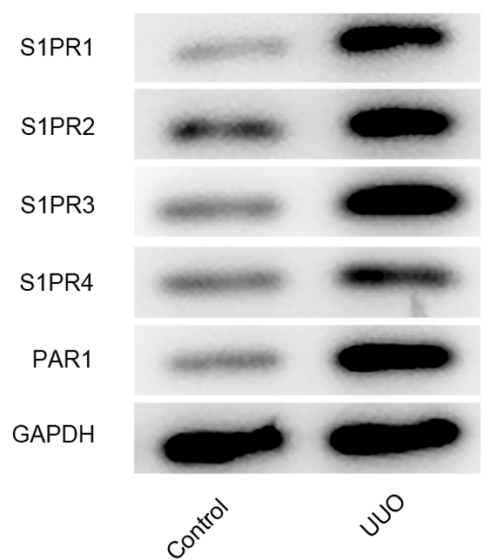

$E$



PAR1

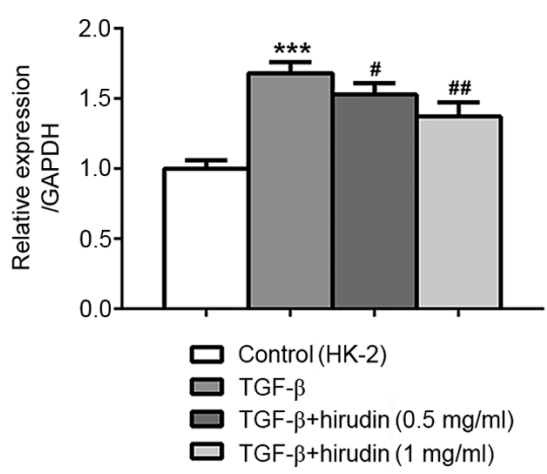

B
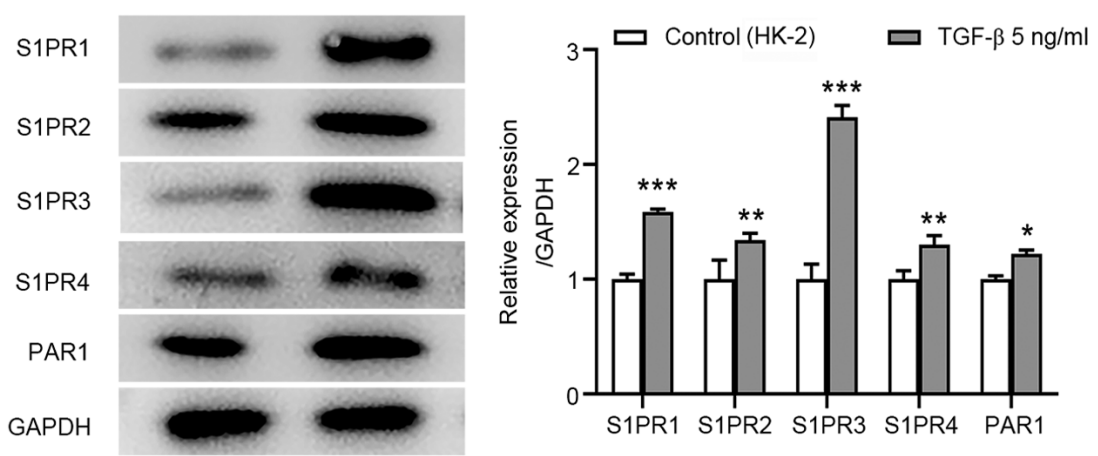

D



$\mathrm{F}$
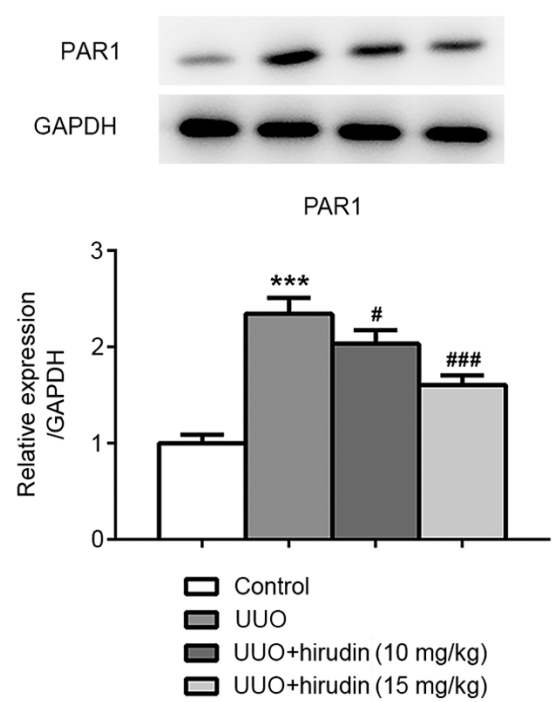

Figure 1. Hirudin inhibits TGF- $\beta$-induced PAR1 upregulation in HK-2 cells and the renal tissues of UUO mice. Relative (A) mRNA levels and (B) protein expression levels of S1PR1-4 and PAR1 in HK-2 cells were detected by RT-qPCR and western blot analysis, respectively. (C) Expression levels of proteins, including S1PR1-4 and PAR1, in the renal tissues of UUO mice were determined by western blotting. (D) Relative mRNA levels of PAR1 in HK-2 cells were determined by RT-qPCR. PAR1 expression in (E) HK-2 cells and (F) renal tissues of UUO mice was quantified by western blotting. Error bars represent the mean \pm SEM from three independent experiments. ${ }^{*} \mathrm{P}<0.05,{ }^{* * *} \mathrm{P}<0.01$ and ${ }^{* * * * *} \mathrm{P}<0.001$ vs. the control. ${ }^{\# P} \mathrm{P}<0.05$, ${ }^{\# \#} \mathrm{P}<0.01$ and ${ }^{\# \# \#} \mathrm{P}<0.001$ vs. TGF- $\beta$ or UUO. UUO, unilateral ureteral ligation; PAR1, protease-activated receptor 1; S1PR, sphingosine-1-phosphate receptor; RT-q, reverse transcription-quantitative.

(1:1,000; cat. no. ab126622; Abcam), S1PR4 (1:1,000; cat. no. ab126392; Abcam), PAR1 (1:1,000; cat. no. 79109; Cell Signaling Technology, Inc.), N-cadherin (1:1,000; cat. no. ab76011; Abcam), slug (1:1,000; cat. no. ab27568; Abcam), E-cadherin (1:1,000; cat. no. ab231303; Abcam), Collagen IV (1:1,000; cat. no. ab227616; Abcam), fibronectin (FN; 1:1,000; cat. no. ab2413; Abcam), MMP9 (1:1,000; cat. no. ab76003; Abcam), MCP-1 (1:500; cat. no. 81559; Cell Signaling
Technology, Inc.) and GAPDH (1:1,000; cat. no. 5174; Cell Signaling Technology, Inc.) at $4^{\circ} \mathrm{C}$ overnight. GAPDH was used as the endogenous control. Following washing three times with TBS $-0.5 \%$ Tween-20, the membrane was incubated with the HRP-conjugated goat anti-rabbit or mouse secondary antibodies (cat. nos. sc-2004 or sc-2005; 1:5,000; Santa Cruz Biotechnology) for $1 \mathrm{~h}$ at room temperature. The protein bands were visualized using ECL reagents (NEN Life Science, Inc.) 
A

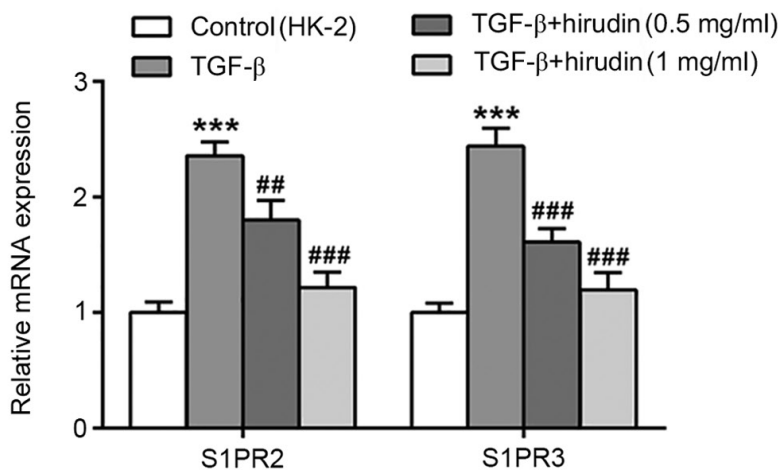

B

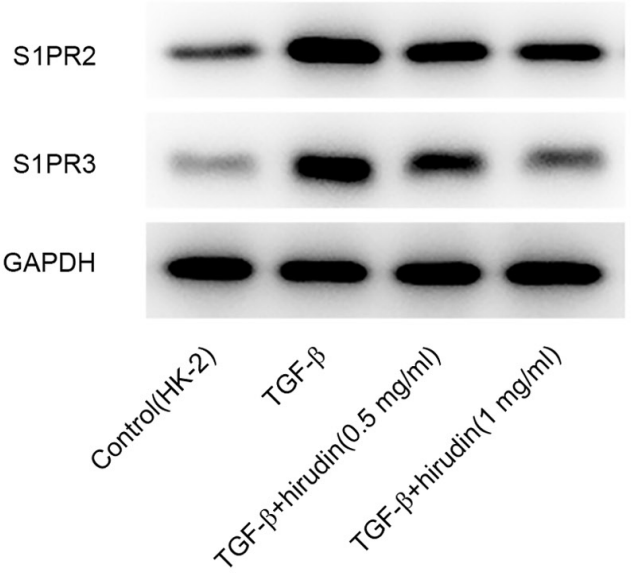

C

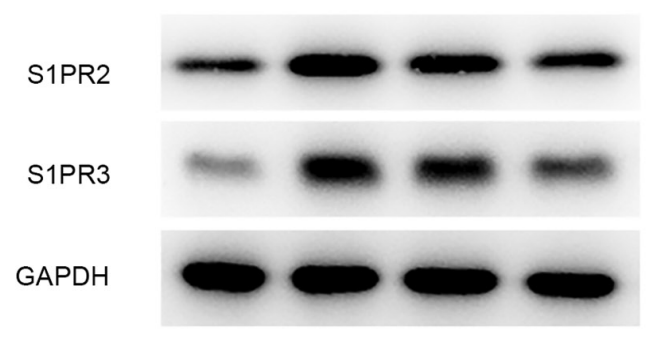

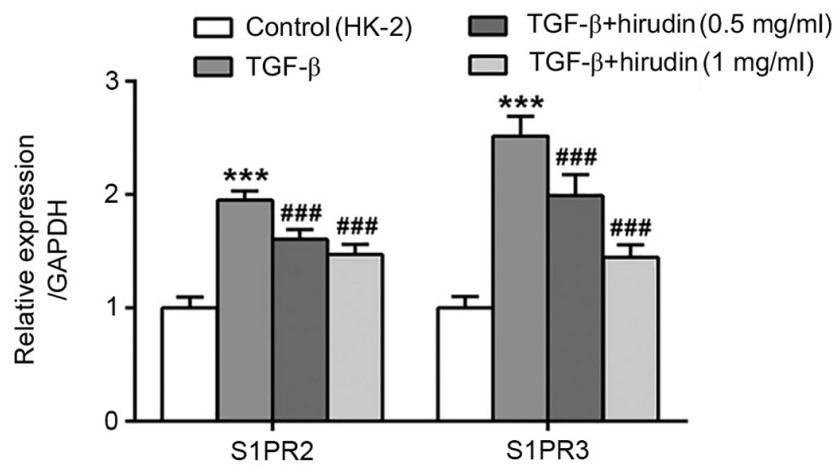

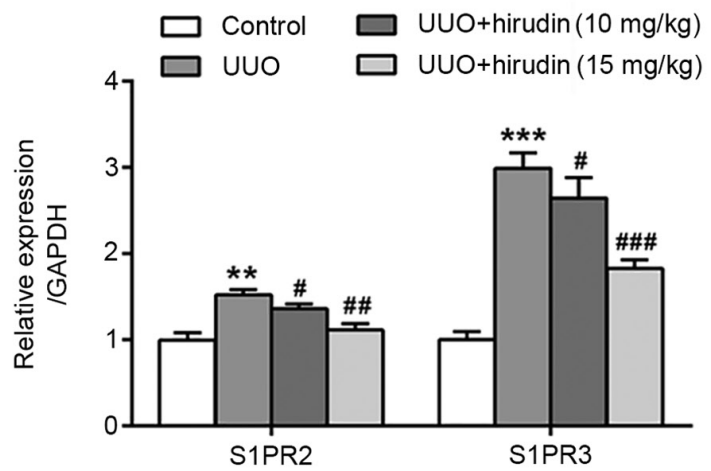

Figure 2. Hirudin inhibits the TGF- $\beta$-induced upregulation of S1PR2 and S1PR3 in both HK-2 cells and renal tissues of UUO mice. (A) Relative mRNA levels of S1PR2 and S1PR3 in HK-2 cells were analyzed by reverse transcription-quantitative PCR. S1PR2 and S1PR3 expression levels in (B) HK-2 cells and (C) renal tissues of UUO mice were quantified by western blotting. Error bars represent the mean \pm SEM from three independent experiments. ${ }^{* *} \mathrm{P}<0.01$ and ${ }^{* * *} \mathrm{P}<0.001$ vs. the control. ${ }^{\#} \mathrm{P}<0.05,{ }^{\# \#} \mathrm{P}<0.01$ and ${ }^{\# \# \#} \mathrm{P}<0.001$ vs. TGF- $\beta$ or UUO. UUO, unilateral ureteral ligation; S1PR, sphingosine-1-phosphate receptor.

and analyzed by densitometry (QuantityOne 4.5.0 software; Bio-Rad Laboratories, Inc.).

Immunofluorescence analysis. HK-2 cells were seeded into 6 -well plates at a density of $5 \times 10^{4}$ cells $/ \mathrm{ml}$, and then treated with the indicated compounds as aforementioned. The cells were washed with PBS and then fixed with $4 \%$ paraformaldehyde for $30 \mathrm{~min}$ at room temperature. Following washing with PBS, cells were blocked with 3\% BSA (Sigma-Aldrich; Merck $\mathrm{KGaA}$ ) for $1 \mathrm{~h}$ at room temperature, and then incubated with primary antibodies against $\alpha$-smooth muscle actin $(\alpha$-SMA; dilution, 1:200; Abcam) at $4^{\circ} \mathrm{C}$ overnight. The cells were then incubated with Alexa Fluor 488-conjugated secondary anti- bodies (dilution, 1:200; cat. no. A0428; Beyotime Institute of Biotechnology) for $1 \mathrm{~h}$ at room temperature, washed with PBS and stained with DAPI. Images were captured under an optical microscope (CKX41; Olympus Corporation; magnification, $\mathrm{x} 200)$.

Statistical analysis. The results are expressed as the mean \pm SEM of at least three independent experiments, and were analyzed using SPSS 18.0 statistical software (SPSS, Inc.). Statistical analysis was performed using unpaired Student's t-test or one-way ANOVA followed by Bonferroni's post hoc test. $\mathrm{P}<0.05$ was considered to indicate a statistically significant difference. 
A



B

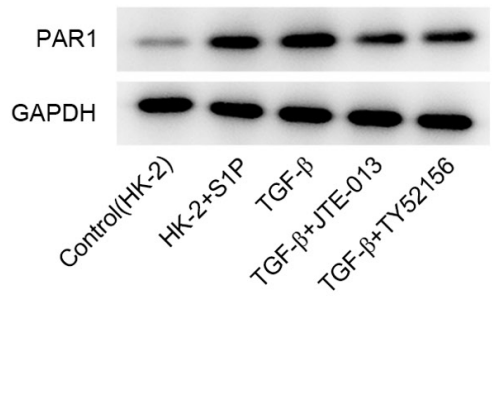

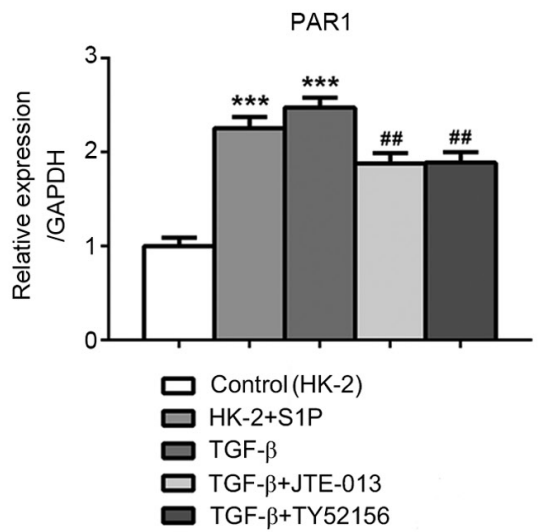

Figure 3. Inhibition of S1PR2 and S1PR3 suppresses PAR1 expression. (A) Relative mRNA level of PAR1 in HK-2 cells was analyzed by reverse transcription-quantitative PCR. (B) PAR1 protein expression in HK-2 cells was quantified by western blotting. Error bars represent the mean \pm SEM from three independent experiments. ${ }^{* * *} \mathrm{P}<0.001$ vs. the control. ${ }^{\# \#} \mathrm{P}<0.01$ and ${ }^{\# \# \#} \mathrm{P}<0.001$ vs. TGF- $\beta$. S1PR, sphingosine-1-phosphate receptor; PAR1, protease-activated receptor 1 .

\section{Results}

S1PR1-4 and PAR1 are upregulated in both TGF- $\beta$-induced $H K-2$ cells and renal tissues from mice with UUO. To determine the pathological mechanism of RIF, the expression levels of S1PR1-4 and PAR1 were evaluated both in vivo and in vitro. RT-qPCR and western blot analyses suggested that the relative mRNA and protein expression levels of S1PR1-4 and PAR1 were increased in HK-2 cells in the TGF- $\beta$ group, compared with the control group (Fig. 1A and B). Additionally, western blot analysis revealed that the protein expression levels of these proteins were also significantly upregulated in renal tissues from mice with UUO, compared with those in normal tissue samples (Fig. 1C). Since both S1PR2 and S1PR3 were markedly upregulated, their roles were further investigated in subsequent experiments.

Hirudin attenuates TGF- $\beta$-mediated PAR1, S1PR2 and SIPR3 upregulation in HK-2 cells and renal tissues from mice with UUO. To investigate the potential mechanism underlying the effects of hirudin on RIF, the expression levels of PAR1, S1PR2 and S1PR3 were further assessed by RT-qPCR and western blot analysis. As shown in Fig. 1D and E, treatment of HK-2 cells with hirudin decreased both the mRNA and protein expression levels of PAR1 in a dose-dependent manner. Consistently, the protein expression level of PAR1 was also downregulated in renal tissues from mice with UUO following treatment with hirudin (Fig. 1F). Additionally, RT-qPCR and western blotting demonstrated that treatment with increasing doses of hirudin dose-dependently attenuated the expression of S1PR2 and S1PR3 at both the mRNA (Fig. 2A) and protein (Fig. 2B) levels in TGF- $\beta$-induced HK-2 cells. Simultaneously, the protein expression levels of S1PR2 and S1PR3 were significantly decreased by hirudin treatment in the renal tissues of mice with UUO (Fig. 2C). The aforementioned findings indicated that hirudin inhibited the TGF- $\beta$-mediated upregulation of PAR1, S1PR2 and S1PR3 both in vivo and in vitro.

Inhibition of SIPR2 and SIPR3 suppresses PAR1 expression. To elucidate the mechanism underlying the effect of hirudin on TGF- $\beta$-induced RIF, PAR 1 expression in TGF- $\beta$-induced
HK-2 cells was detected (20). RT-qPCR and western blotting revealed that treatment with $\mathrm{S} 1 \mathrm{P}$ and TGF- $\beta$ significantly upregulated PAR1, and the increased PAR1 level was partially restored following treatment with JTE-013 or TY52156 (Fig. 3A and B). These results suggested that S1P could promote PAR1 expression.

Hirudin attenuates TGF- $\beta$-induced EMT, fibrosis and MCP-1 expression through S1P/S1PR2/S1PR3 signaling-mediated $P A R 1$ downregulation. To further verify the molecular mechanism underlying the effect of hirudin on TGF- $\beta$-induced fibrosis, HK-2 cells were treated with PAR1 agonists (TFL; $50 \mu \mathrm{M}$ ) for $2 \mathrm{~h}$. As shown in Fig. 4A, western blotting revealed that hirudin treatment significantly decreased the TGF- $\beta$-mediated upregulation of $\mathrm{N}$-cadherin and Slug, whilst restoring TGF- $\beta$-mediated E-cadherin downregulation. Notably, exposure to S1P and TFL reversed the effect of hirudin on the expression of EMT-related proteins. The immunofluorescence assay results showed that hirudin significantly attenuated TGF- $\beta$-induced $\alpha$-SMA upregulation, which was partially inhibited by S1P and TFL agonists (Fig. 4B and C). Additionally, western blotting demonstrated that hirudin significantly abrogated the TGF- $\beta$-mediated upregulation of fibrosis-related proteins, including collagen IV, FN and MMP9. Furthermore, exposure to S1P and TFL also reversed the effect of hirudin on the expression of fibrosis-related proteins (Fig. 5A). Finally, western blotting and RT-qPCR demonstrated that hirudin treatment notably decreased TGF- $\beta$-induced MCP-1 protein and mRNA expression levels, respectively. By contrast, treatment with S1P and TFL restored the expression of MCP-1, even when combined with hirudin (Fig. 5B and C), thus suggesting that hirudin attenuated TGF- $\beta$-induced EMT, fibrosis and MCP-1 expression through PAR1 inhibition mediated via the S1P/S1PR2/S1PR3 signaling pathway.

\section{Discussion}

RIF is defined as a chronic and progressive process, ultimately resulting in loss of renal function during aging and CKD progression. Since RIF has a negative impact on patient quality of life, it is necessary to determine effective 
A
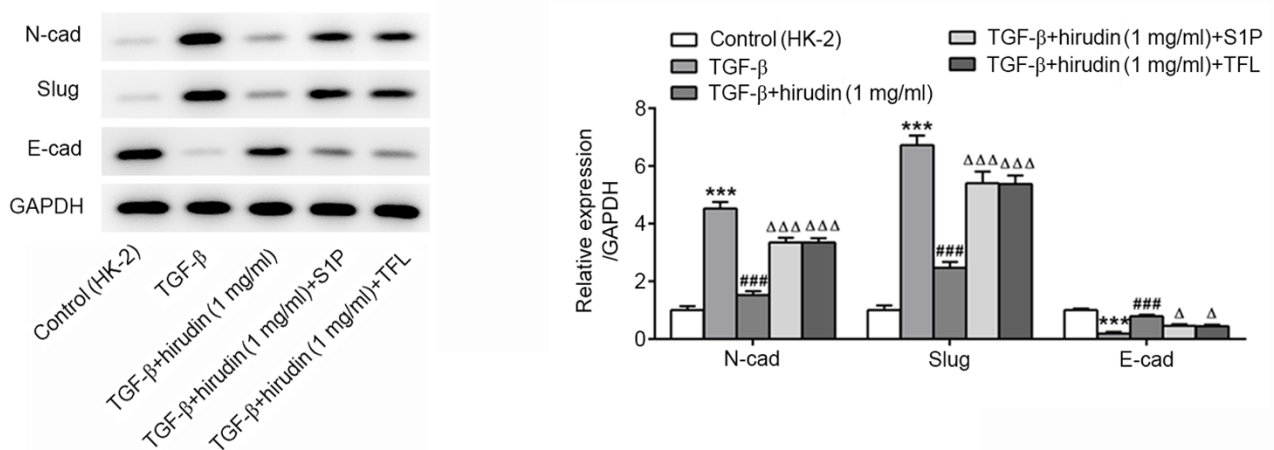

TGF- $\beta$ +hirudin (1 mg/ml) TGF- $\beta$ +hirudin (1 mg/ml)
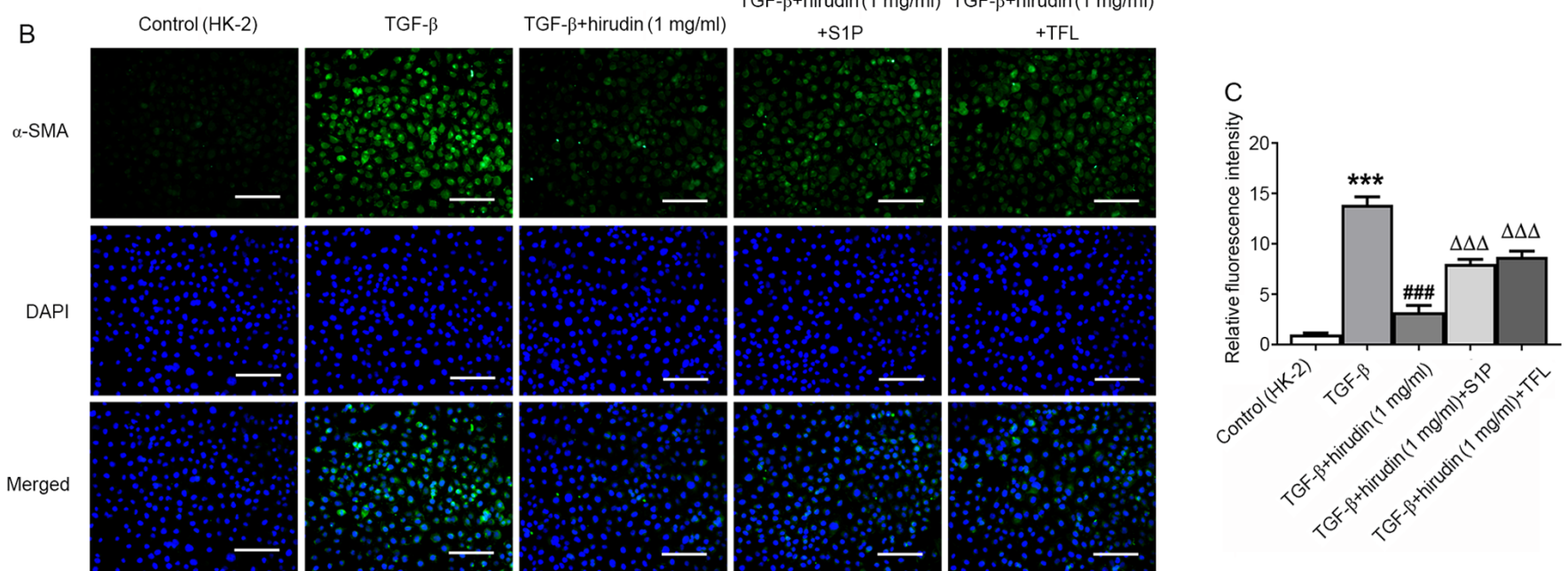

Figure 4. Hirudin suppresses TGF- $\beta$-induced epithelial-mesenchymal transition via S1P/S1PR2/S1PR3 signaling-mediated PAR1 inhibition. (A) Expression levels of N-cad, Slug and E-cad in HK-2 cells were determined using western blotting. (B and C) $\alpha$-SMA expression in HK-2 cells was analyzed by immunofluorescence analysis. Scale bar, $100 \mu \mathrm{m}$. Error bars represent the mean \pm SEM from three independent experiments. ${ }^{* * *} \mathrm{P}<0.001$ vs. the control. ${ }^{\# \# "} \mathrm{P}<0.001$ vs. TGF- $\beta$. ${ }^{\Delta \mathrm{P}}<0.05$ and ${ }^{\Delta \Delta \Delta} \mathrm{P}<0.001$ vs. TGF- $\beta+$ hirudun $(1 \mathrm{mg} / \mathrm{ml})$. S1PR, sphingosine-1-phosphate receptor; PAR1, protease-activated receptor 1; N-cad, $\mathrm{N}$-cadherin; E-cad, E-cadherin; $\alpha$-SMA, $\alpha$-smooth muscle actin; S1P, sphingosine-1-phosphate; TFL, TFLLR-NH2.

therapeutic strategies for RIF. Our previous study demonstrated that hirudin attenuated fibrosis in renal tissues from mice with UUO, as well as inhibiting inflammation, EMT and apoptosis in HK-2 renal tubular epithelial cells (16). Therefore, the present study aimed to further verify the effect of hirudin on RIF, and to elucidate the potential mechanism underlying its effect.

Diabetes and hypertension are the two major risk factors for CKD. It has been reported that the expression level of the fibrogenic cytokine TGF- $\beta$ is upregulated under hyperglycemic conditions (21). Herein, HK-2 cells were stimulated with TGF- $\beta$ to establish an in vitro RIF cell model. The results demonstrated that the expression levels of S1PR1-4 and PAR1 were significantly increased in both TGF- $\beta$-induced HK- 2 cells and renal tissues from mice with UUO, particularly those of S1PR2 and S1PR3. Natural products have been widely reported to protect against TGF- $\beta$-induced renal fibrosis (22-24). As one extract of natural products, hirudin abrogated TGF- $\beta$-induced PAR1, S1PR2 and S1PR3 upregulation in both HK-2 cells and murine renal tissues. A previous study demonstrated that the TGF- $\beta$ /sphingosine kinase $1 / \mathrm{S} 1 \mathrm{P}$ signaling pathway promoted fibrosis in renal tubular epithelial cells (25), which was consistent with the results of the current study. Furthermore, the inactivation of S1P/S1PR2 signaling ameliorated diabetic nephropathy by restoring high glucose-mediated intercellular adhesion molecule 1 and FN upregulation in glomerular mesangial cells (26). Moreover, S1P upregulated the expression of PAR1 and PAR4 (18). Herein, the inhibition of S1PR2 and S1PR3 markedly suppressed PAR1 expression. The aforementioned findings suggested that hirudin could ameliorate TGF- $\beta$-induced RIF by PAR1 inhibition via the S1P/S1PR2/ S1PR3 signaling pathway. However, the specific constituent of the S1P/S1PR2/S1PR3 pathway responsible for these effects was not determined in the present study, and the regulatory effects of S1P/S1PR2/S1PR3 signaling in renal fibrosis (and the primary modulator therein) will be investigated in future studies.

To further verify whether PAR1 and S1P/S1PR2/S1PR3 signaling could mediate the effect of hirudin on RIF development, the expression levels of EMT- and fibrosis-related proteins were determined by western blot analysis. EMT is characterized by E-cadherin downregulation, and $\mathrm{N}$-cadherin and Slug upregulation (27). Additionally, $\alpha$-SMA, collagen-IV, FN and MMP9 are considered as reliable markers of EMT in various organs during fibrosis (28). Therefore, EMT plays an essential role in the pathogenesis of renal fibrosis. Herein, hirudin significantly decreased the expression levels of $\mathrm{N}$-cadherin, Slug, $\alpha$-SMA, collagen-IV, FN and MMP9, and 
A

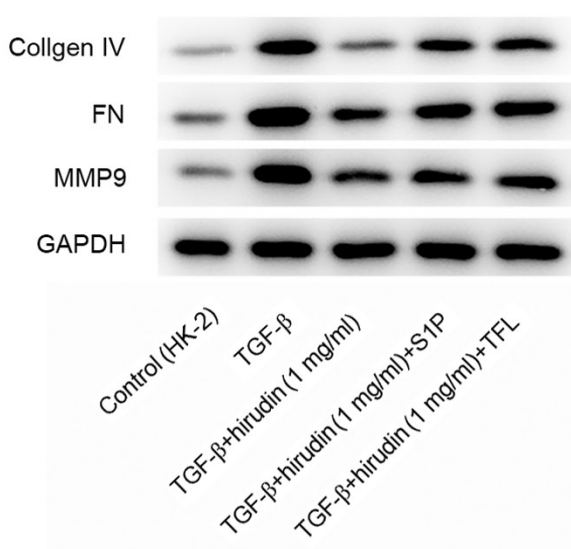

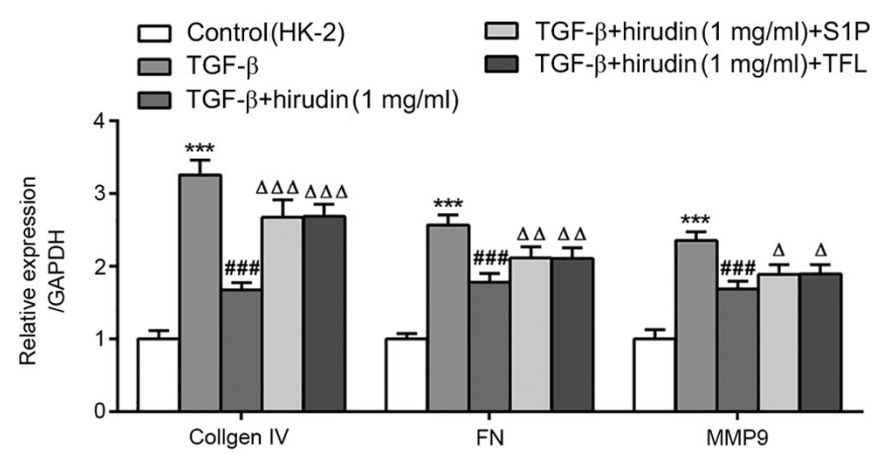

B

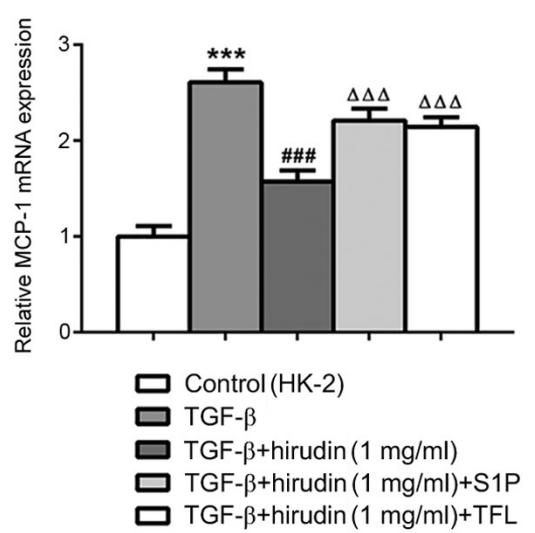

C

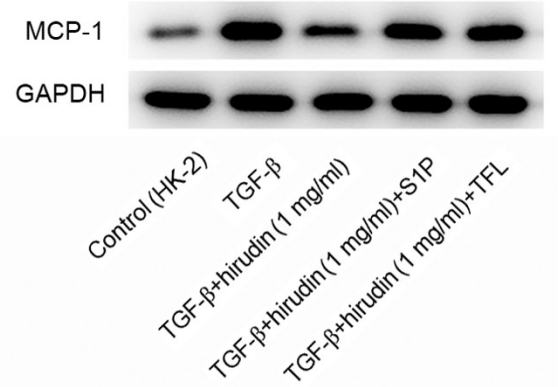

MCP-1



Figure 5. Hirudin suppresses TGF- $\beta$-induced fibrosis and MCP-1 expression via S1P/S1PR2/S1PR3 signaling-mediated PAR1 inhibition. (A) Expression levels of collagen IV, FN and MMP-9 in HK-2 cells were determined by western blotting. (B) Relative mRNA level of MCP-1 in HK-2 cells was analyzed by reverse transcription-quantitative PCR. (C) MCP-1 expression in HK-2 cells was quantified by western blotting. Error bars represent the mean \pm SEM from three independent experiments. ${ }^{* * *} \mathrm{P}<0.001$ vs. the control. ${ }^{\# \# \#} \mathrm{P}<0.001$ vs. TGF- $\beta .{ }^{\Delta} \mathrm{P}<0.05,{ }^{\Delta \Delta} \mathrm{P}<0.01$ and ${ }^{\Delta \Delta \Delta} \mathrm{P}<0.001$ vs. TGF- $\beta+h i r u d u n(1 \mathrm{mg} / \mathrm{ml})$. MCP-1, monocyte chemoattractant protein 1; S1PR, sphingosine-1-phosphate receptor; PAR1, protease-activated receptor 1; FN, fibronectin; S1P, sphingosine-1-phosphate; TFL, TFLLR-NH2.

increased that of E-cadherin. Of note, S1P and PAR1 agonists abolished the effects of hirudin on the expression of EMT- and fibrosis-related proteins.

Renal fibrosis is caused by the imbalance between the excessive synthesis and reduced breakdown of ECM in the renal parenchyma in response to injury and inflammation, eventually resulting in the loss of renal function. The activation of PAR1 can be induced by thrombin, which promotes proinflammatory responses in endothelial cells (29). A previous study showed that stimulation of renal tubular epithelial cells with TGF- $\beta$ promoted MCP-1 upregulation (30). Furthermore, PAR1 deletion resulted in diminished production of MCP-1 in renal tissues from mice with UUO (31). In the present study, hirudin significantly downregulated MCP-1, which was reversed following treatment with S1P and PAR1 agonists. Therefore, hirudin may suppress TGF- $\beta$-induced EMT, fibrosis and MCP-1 expression via the S1P/S1PR2/ S1PR3 signaling-mediated inhibition of PAR1. Taken together, the aforementioned results indicated that hirudin attenuated TGF- $\beta$-induced fibrosis in renal proximal tubular epithelial cells by downregulating PAR1 via S1P/S1PR2/S1PR3 signaling.
In summary, the current study revealed that hirudin inhibited TGF- $\beta$-induced PAR1, S1PR2 and S1PR3 upregulation in both HK-2 cells and renal tissues from mice with UUO. Additionally, inhibition of S1PR2 and S1PR3 suppressed the expression of PAR1. Collectively, hirudin attenuated TGF- $\beta$-induced EMT, fibrosis and MCP-1 expression via PAR1 inhibition mediated by S1P/S1PR2/S1PR3 signaling, suggesting that hirudin administration may be a promising effective strategy for RIF treatment.

\section{Acknowledgements}

Not applicable.

\section{Funding}

The present study was supported by National Natural Science Foundation of China (grant no. 82060804), Guangxi University of Chinese Medicine Science Research Program (grant no. 2020MS022), the General Program of the Guangxi Natural Science Foundation (grant no. 2020GXNSFAA259086) and the Guangxi Traditional Chinese Medicine Appropriate 
Technology Development and Promotion Program (grant no. GZSY20-27).

\section{Availability of data and materials}

The datasets used and/or analyzed during the current study are available from the corresponding author on reasonable request.

\section{Authors' contributions}

QL and YX designed the experiments and wrote the manuscript. QL, CL, ZW, RW and JM performed the experiments. WS and JQ analyzed the data. JM searched the literature. All authors have read and approved the final manuscript. YX and JM confirm the authenticity of all the raw data.

\section{Ethics approval and consent to participate}

All animal experiments were approved by the Guangxi University of Chinese Medicine Institutional Animal Ethics and Welfare Committee (approval no. DW20190107-013).

\section{Patient consent for publication}

Not applicable.

\section{Competing interests}

The authors declare that they have no competing interests.

\section{References}

1. Martínez-Klimova E, Aparicio-Trejo OE, Tapia E and Pedraza-Chaverri J: Unilateral ureteral obstruction as a model to investigate fibrosis-attenuating treatments. Biomolecules 9: 9, 2019.

2. Wen Y, Rudemiller NP, Zhang J, Jeffs AD, Griffiths R, Lu X, Ren J, Privratsky J and Crowley SD: Stimulating type 1 angiotensin receptors on T lymphocytes attenuates renal fibrosis. Am J Pathol 189: 981-988, 2019.

3. Li R, Guo Y, Zhang Y, Zhang X, Zhu L and Yan T: Salidroside ameliorates renal interstitial fibrosis by inhibiting the TLR4/ NF- $\mathrm{B}$ and MAPK signaling pathways. Int J Mol Sci 20: 1103, 2019.

4. Lv W, Fan F, Wang Y, Gonzalez-Fernandez E, Wang C, Yang L, Booz GW and Roman RJ: Therapeutic potential of microRNAs for the treatment of renal fibrosis and CKD. Physiol Genomics 50: 20-34, 2018.

5. Nastase MV, Zeng-Brouwers J, Wygrecka M and Schaefer L: Targeting renal fibrosis: Mechanisms and drug delivery systems. Adv Drug Deliv Rev 129: 295-307, 2018.

6. Humphreys BD: Mechanisms of renal fibrosis. Annu Rev Physiol 80: 309-326, 2018.

7. Chen Y, Mu L, Xing L, Li S and Fu S: Rhein alleviates renal interstitial fibrosis by inhibiting tubular cell apoptosis in rats. Biol Res 52: 50, 2019.

8. Nogueira A, Pires MJ and Oliveira PA: Pathophysiological mechanisms of renal fibrosis: A review of animal models and therapeutic strategies. In Vivo 31: 1-22, 2017.

9. Ma Z, Wei Q, Zhang M, Chen JK and Dong Z: Dicer deficiency in proximal tubules exacerbates renal injury and tubulointerstitial fibrosis and upregulates Smad2/3. Am J Physiol Renal Physiol 315: F1822-F1832, 2018.

10. Forbes MS, Thornhill BA, Minor JJ, Gordon KA, Galarreta CI and Chevalier RL: Fight-or-flight: Murine unilateral ureteral obstruction causes extensive proximal tubular degeneration, collecting duct dilatation, and minimal fibrosis. Am J Physiol Renal Physiol 303: F120-F129, 2012.

11. Isaka Y: Targeting TGF- $\beta$ signaling in kidney fibrosis. Int J Mol Sci 19: 19, 2018.
12. Wang J, Zhu H, Huang L, Zhu X, Sha J, Li G, Ma G, Zhang W, Gu M and Guo Y: Nrf2 signaling attenuates epithelial-to-mesenchymal transition and renal interstitial fibrosis via PI3K/Akt signaling pathways. Exp Mol Pathol 111: 104296, 2019.

13. Wakui M, Fujimori Y, Nakamura S, Kondo Y, Kuroda Y, Oka S, Nakagawa T, Katagiri H and Murata M: Distinct features of bivalent direct thrombin inhibitors, hirudin and bivalirudin, revealed by clot waveform analysis and enzyme kinetics in coagulation assays. J Clin Pathol 72: 817-824, 2019.

14. Deng F, Zhang J, Li Y, Wang W, Hong D, Li G and Feng J: Hirudin ameliorates immunoglobulin A nephropathy by inhibition of fibrosis and inflammatory response. Ren Fail 41: 104-112, 2019.

15. Xie Y, Lan F, Zhao J and Shi W: Hirudin improves renal interstitial fibrosis by reducing renal tubule injury and inflammation in unilateral ureteral obstruction (UUO) mice. Int Immunopharmacol 81: 106249, 2020.

16. Bae JS, Kim IS and Rezaie AR: Thrombin down-regulates the TGF-beta-mediated synthesis of collagen and fibronectin by human proximal tubule epithelial cells through the EPCR-dependent activation of PAR-1. J Cell Physiol 225: 233-239, 2010.

17. Sun XJ, Chen M and Zhao MH: Thrombin contributes to anti-myeloperoxidase antibody positive IgG-mediated glomerular endothelial cells activation through SphK1-S1P-S1PR3 signaling. Front Immunol 10: 237, 2019.

18. Mahajan-Thakur S, Sostmann BD, Fender AC, Behrendt D, Felix SB, Schrör K and Rauch BH: Sphingosine-1-phosphate induces thrombin receptor PAR-4 expression to enhance cell migration and COX-2 formation in human monocytes. J Leukoc Biol 96: 611-618, 2014.

19. Wang GL, Xia XL, Li XL, He FH and Li JL: Identification and expression analysis of the MSP130-related-2 gene from Hyriopsis cumingii. Genet Mol Res 14: 4903-4913, 2015.

20. Hirata N, Yamada S, Shoda T, Kurihara M, Sekino Y and Kanda Y: Sphingosine-1-phosphate promotes expansion of cancer stem cells via S1PR3 by a ligand-independent Notch activation. Nat Commun 5: 4806, 2014.

21. Hsieh PF, Liu SF, Lee TC, Huang JS, Yin LT, Chang WT, Chuang LY, Guh JY, Hung MY and Yang YL: The role of IL-7 in renal proximal tubule epithelial cells fibrosis. Mol Immunol 50: 74-82, 2012.

22. Chen HA, Chen CM, Guan SS, Chiang CK, Wu CT and Liu SH: The antifibrotic and anti-inflammatory effects of icariin on the kidney in a unilateral ureteral obstruction mouse model. Phytomedicine 59: 152917, 2019.

23. Chen DQ, Cao G, Zhao H, Chen L, Yang T, Wang M, Vaziri ND, Guo Y and Zhao YY: Combined melatonin and poricoic acid A inhibits renal fibrosis through modulating the interaction of Smad 3 and $\beta$-catenin pathway in AKI-to-CKD continuum. Ther Adv Chronic Dis 10: 2040622319869116, 2019.

24. Chen DQ, Feng YL, Cao G and Zhao YY: Natural products as a source for antifibrosis therapy. Trends Pharmacol Sci 39: 937-952, 2018.

25. Liu X, Hong Q, Wang Z, Yu Y, Zou X and Xu L: Transforming growth factor- $\beta$-sphingosine kinase $1 / \mathrm{S} 1 \mathrm{P}$ signaling upregulates microRNA-21 to promote fibrosis in renal tubular epithelial cells. Exp Biol Med (Maywood) 241: 265-272, 2016.

26. Yang Z, Xiong F, Wang Y, Gong W, Huang J, Chen C, Liu P and Huang H: TGR5 activation suppressed S1P/S1P2 signaling and resisted high glucose-induced fibrosis in glomerular mesangial cells. Pharmacol Res 111: 226-236, 2016.

27. Man XY, Chen XB, Li W, Landeck L, Dou TT, Chen JQ, Zhou J, Cai SQ and Zheng M: Analysis of epithelial-mesenchymal transition markers in psoriatic epidermal keratinocytes. Open Biol 5: $150032,2015$.

28. Xu R, Chen MY, Liang W, Chen Y and Guo MY: Zinc deficiency aggravation of ROS and inflammatory injury leading to renal fibrosis in mice. Biol Trace Elem Res 199: 622-632, 2021.

29. Posma JJ, Grover SP, Hisada Y, Owens AP III, Antoniak S, Spronk HM and Mackman N: Roles of coagulation proteases and PARs (protease-activated receptors) in mouse models of inflammatory diseases. Arterioscler Thromb Vasc Biol 39: 13-24, 2019.

30. Liang D, Song Z, Liang W, Li Y and Liu S: Metformin inhibits TGF-beta 1-induced MCP-1 expression through BAMBI-mediated suppression of MEK/ERK1/2 signalling. Nephrology (Carlton) 24: 481-488, 2019.

31. Waasdorp M, de Rooij DM, Florquin S, Duitman J and Spek CA: Protease-activated receptor-1 contributes to renal injury and interstitial fibrosis during chronic obstructive nephropathy. J Cell Mol Med 23: 1268-1279, 2019.

This work is licensed under a Creative Commons Attribution-NonCommercial-NoDerivatives 4.0 International (CC BY-NC-ND 4.0) License. 